
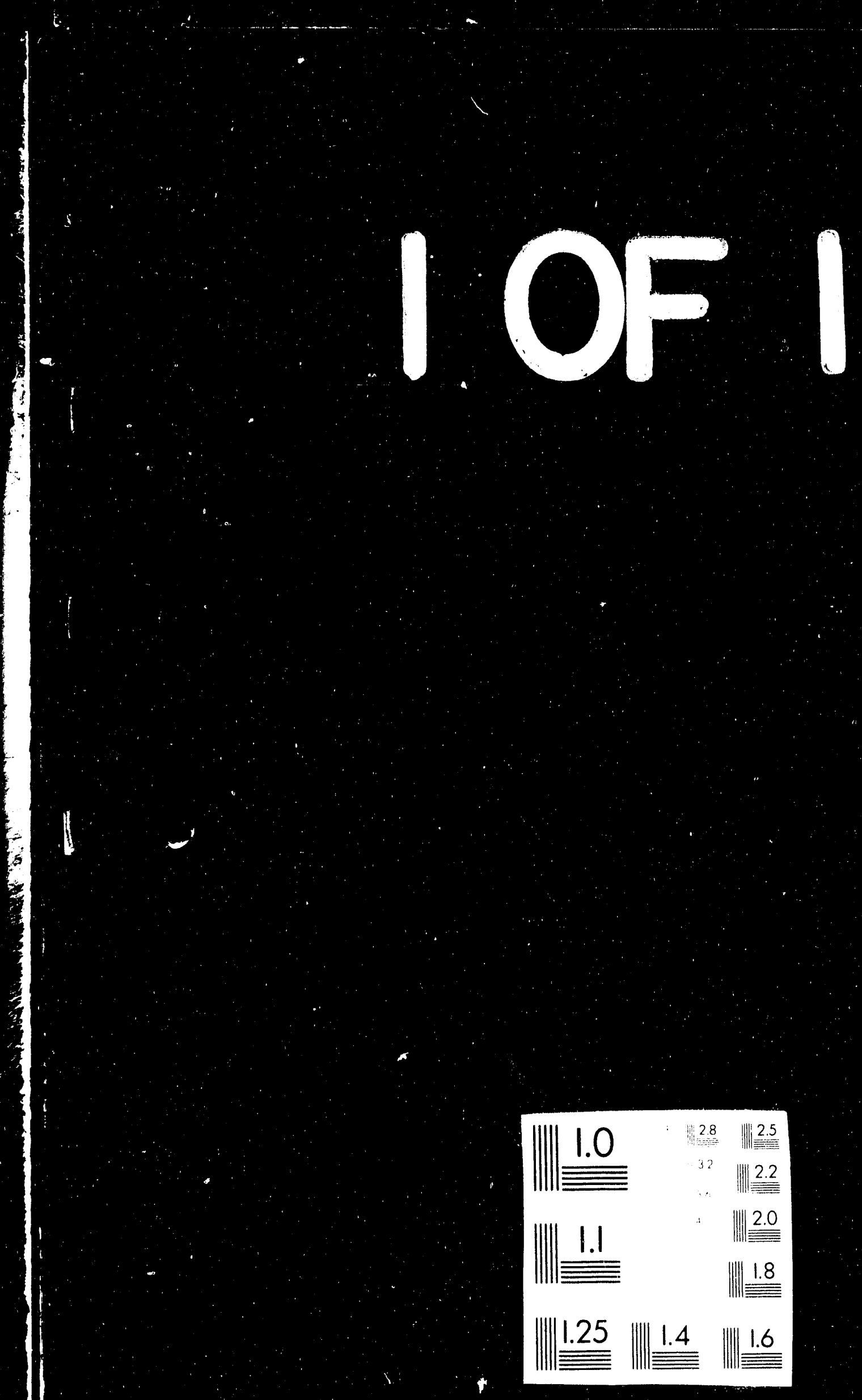


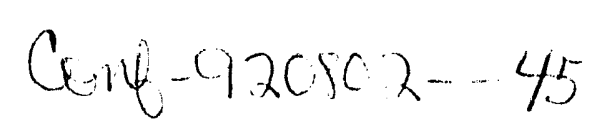

LBL-32891

UC-404

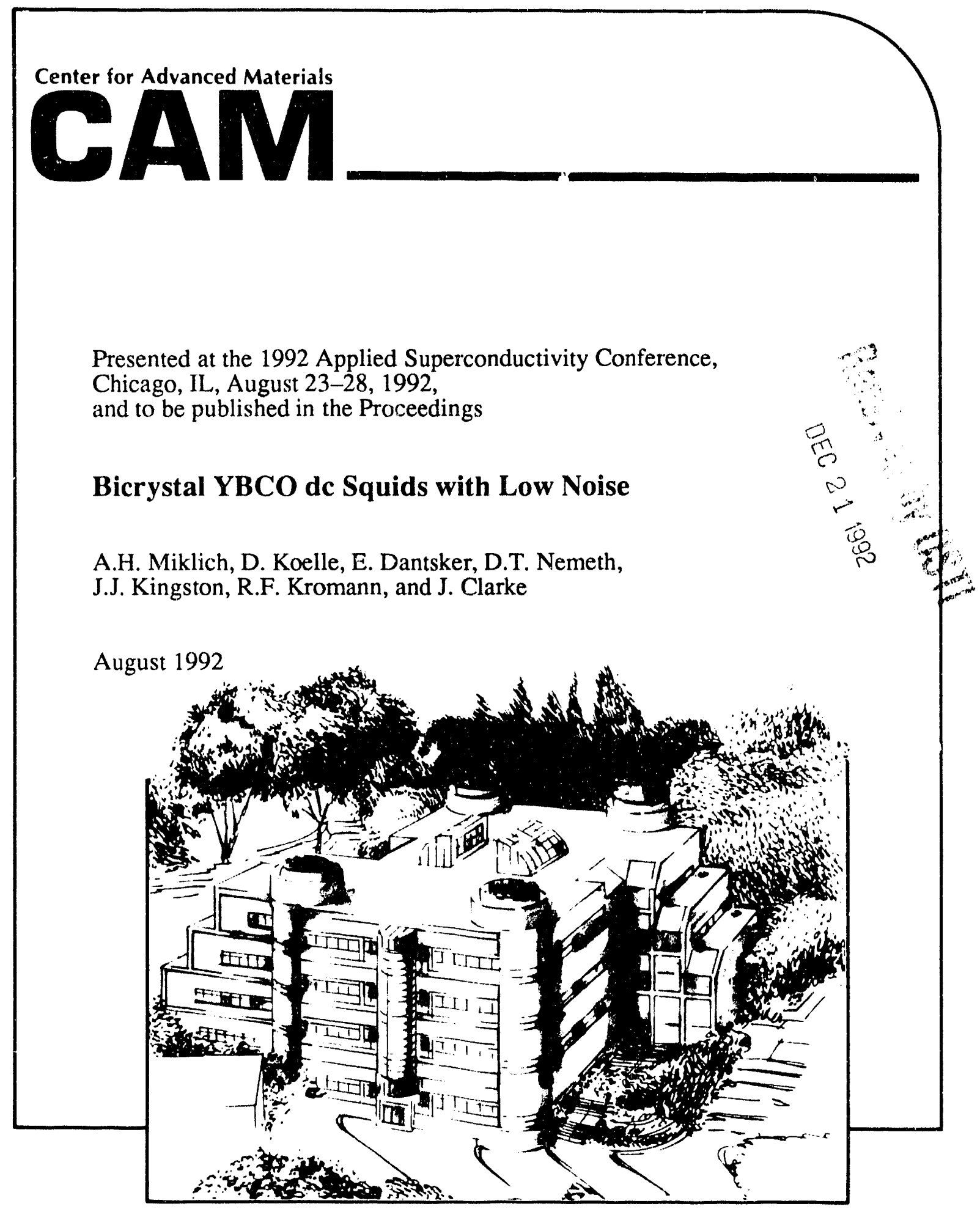

\section{Materials and Chemical Sciences Division}

\section{Lawrence Berkeley Laboratory - University of California}

ONE CYCLOTRON ROAD, BERKELEY, CA 94720 •(415) 486-4755 


\section{DISCLAIMER}

This document was prepared as an account of work sponsoned by the United States Government. Neither the United States Government nor any agency thereof, nor The Regents of the University of California, nor any of their employees, makes any warranty, express or implied, or assumes any legal liability or responsibility for the accuracy, completeness, or usefulness of any information, apparatus, product, or process disclosed, or represents that its use would not infringe privately owned rights. Reference herein to any specific commercial product, process, or service by its trade name, trademark, manufacturer, or otherwise, does not necessarily constitute or imply its endorsement, recommendation, or favoring by the United States Government or any agency thereof, or The Regents of the University of California. The views and opinions of authors expressed herein do not necessarily state or reflect those of the United States Government or any agency thereof or The Regents of the University of Califomia and shall not be used for advertising or product endorsement purposes.

Lawrence Berkeley Laboratory is an equal opportunity employer. 
LBL- -32891

DE93 004779

\section{BICRYSTAL YBCO DC SQUIDS WITH LOW NOISE}

A. H. Miklich, D. Koelle, E. Dantsker, D. T. Nemeth, J. J. Kingston, R. F. Kromann and J. Clarke

Department of Physics, University of California,

Center for Advanced Materials, Materials Sciences Division, Lawrence Berkeley Laboratory,

Berkeley, CA 94720

This work was supported by the California Competitive Technology Program and by the Director, Office of Energy Research, Office of Basic Energy Sciences, Materials Sciences Division of the U.S. Department of Energy under contract number DE-AC03-76SF00098.
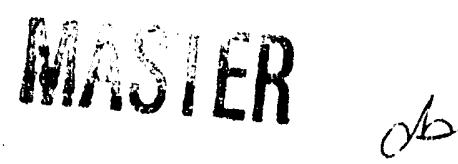


\title{
BICRYSTAL YBCO DC SQUIDS WITH LOW NOISE
}

\author{
A. H. Miklich, D. Koelle, E. Dantsker, D. T. Nemeth, J. J. Kingston, R. F. Kromann and J. Clarke \\ Department of Physics, University of Califomia, \\ Center for Advanced Materials, Materials Sciences Division, \\ Lawrence Berkeley Laboratory, \\ Berkeley, CA 94720
}

\begin{abstract}
We have fabricated $12 \mathrm{dc}$ SQUIDs by laser depositing $\mathrm{YBa}_{2} \mathrm{Cu}_{3} \mathrm{O}_{7-x}$ on a $\mathrm{SrTiO}_{3}$ bicrystal substrate with a misorientation angle of $24^{\circ}$. At $77 \mathrm{~K}$ all twelve devices had acceptable values of critical current, resistance and voltage modulation produced by an external magnetic field. The white noise energy of one device with an estimated inductance of $41 \mathrm{pH}$ was $1.8 \times 10^{-30} \mathrm{JHz}^{-1}$. The noise power scaled as $\mathrm{V} / \mathrm{f}$ at frequencies below about $1 \mathrm{kHz}$, however; by using a bias current reversal scheme we were able to reduce this noise by two orders of magnitude at $1 \mathrm{~Hz}$, to a value of about $1.5 \times$ $10^{-29} \mathrm{JHz}^{-1}$. We made a magnetometer by coupling the SQUID to a flux transformer with a 5-turn input coil. The measured magnetic field gain was 60 , and the white noise $36 \mathrm{fT} \mathrm{Hz}-1 / 2$. However, the transformer produced relatively large levels of $1 / f$ flux noise, not reduced by the bias reversal scheme, that limited the noise at $1 \mathrm{~Hz}$ to $1.7 \mathrm{pT} \mathrm{Hz}^{-1 / 2}$. A single-layer magnetometer with a single-tum pick up loop is briefly described.
\end{abstract}

\section{INTRODUCTION}

There has recently been considerable progress in the development of Superconducting QUantum Interference Devices (SQUIDs) fabricated from thin films of high transition temperature $\left(T_{C}\right)$ superconductors. [1-6] Of particular interest for many applications is the level of $\mathrm{y} / \mathrm{f}$ noise at low frequencies $f$. This noise may be inherent to the junctions where it arises from critical current fluctuations, $[4,7,8]$ or it may arise from the motion of vortices trapped in the body of the SQUID. [9] It is possible to reduce the contribution of the first mechanism substantially by applying one of several electronic modulation schemes to the dc SQUID, whereas the latter mechanism cannot be reduced in this way. In this paper, we report a study of YBCO SQUIDs deposited on a single bicrystal substrate. We describe the reduction of the low frequency $1 / \mathrm{f}$ noise by an electronic modulation scheme to a level that is technologically useful. Finally, we describe magnetometers involving both mulatum flux transformers and single-loop devices fabricated in a single layer.

This work was supported by the California Competitive Technology Program and by the Director, Office of Energy Research, Office of Basic Energy Sciences, Materials Sciences Division of the U.S. Department of Energy under contract number DE-AC03-76SF00098. A. H. Miklich was supported by the Fannie and John Hertz Foundation. D. Koelle acknowledges support by the Deutsche Forschungs Gemeinschaft.

Manuscript received August 24, 1992.

\section{FABRICATION}

We use a pulsed excimer laser to deposit YBCO films 200 to $300 \mathrm{~nm}$ thick on $10 \mathrm{~mm} \times 10 \mathrm{~mm}(100) \mathrm{SrTiO}_{3}$ bicrystal substrates with a $24^{\circ}$ misorientation angle. [10] Subsequently, we laser deposit an $\mathrm{Ag}$ film to a thickness of $100-200 \mathrm{~nm}$ through a shadow mask over the part of the YBCO film to be used for contact pads. The faint line of YBCO grains along the grain boundary of the substrate allows us to align a mask for photolithographic patterning. We first ion mill the Ag through the photomask and then etch the SQUID in $0.05-0.1 \%$ nitric acid. The acid undercuts the photoresist in a controllable way, enabling us to make a bridge $2-4 \mu \mathrm{m}$ wide for the junctions. Finally, we make electrical connections to the SQUIDs by ultrasonic bonding $25 \mu \mathrm{m}$ diameter Al wires to the Ag-covered contact pads.

Figure 1 shows the configuration of two types of squarewasher SQUIDs; both have inner holes of $25 \mu \mathrm{m} \times 25 \mu \mathrm{m}$. The type $A$ washers have an outer dimension of $250 \mu \mathrm{m} \times$ $250 \mu \mathrm{m}$, while the type B were either $250 \mu \mathrm{m} \times 250 \mu \mathrm{m}$ or $500 \mu \mathrm{m} \times 500 \mathrm{~mm}$. In the type $A$ design, the junctions are placed outside the washer, to maintain a high coupling efficiency to a multiturn input coil. However, the extra inductance associated with the slit reduces the signal available from the SQUID. In the type B design, the junctions are placed at the inner edge of the washer, thereby eliminating the inductance of the slit. On the other hand, the junctions are now in the relatively high magnetic field region produced by
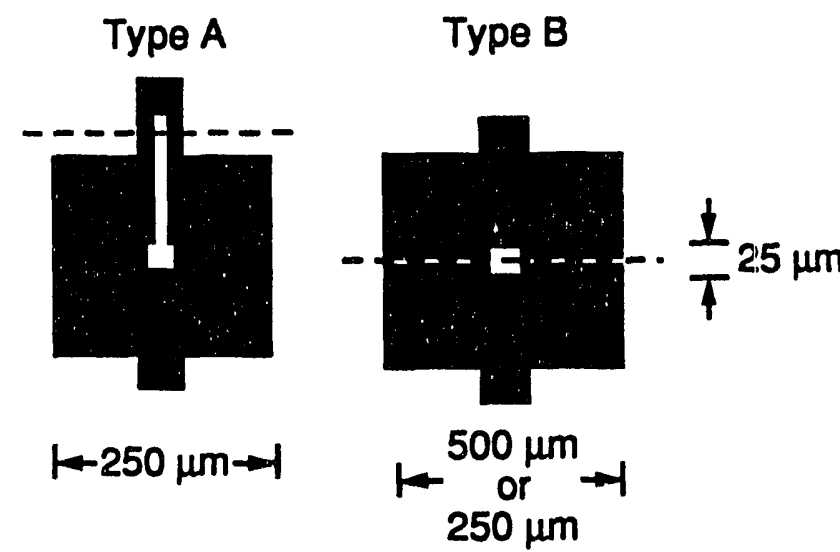

Fig. 1 Two types of YBCO SQUID fabricated on a $\mathrm{SrTiO}_{3}$ bicrystal with grain boundary shown with dashed line. Junction widths were $2-4 \mu \mathrm{m}$. 
the flux focusing [11] of the SQUID body, which introduces additional single-junction modulation effects in the earth's field. We tested 9 type A SQUIDs and 3 type B SQUIDs on one chip.

\section{RESULTS}

We first studied the properties of our SQUIDs by cooling them, in the earth's magnetic field, in liquid nitrogen. The current-voltage (I-V) characteristics were close to those expected from the resistively shunted junction model. Figure 2 summarizes the critical currents $\left(2 \mathrm{I}_{\mathcal{c}}\right)$, maximized by rotating the probe in the ambient magnetic field, and asymptotic resistances $\left(R_{N} / 2\right)$ for all 12 SQUIDs on a single chip vith nominally identical junction widths of $3 \mu \mathrm{m} ; \mathrm{I}_{\mathrm{c}}$ and $\mathrm{R}_{\mathrm{N}}$ refer to single junctions. Also shown is the maximum peak-to-peak voltage modulation, $\Delta \mathrm{V}$, obtained when the magnetic flux is varied at the optimum bias current. The average values of these parameters are $2 \mathrm{I}_{\mathrm{c}}=185 \mu \mathrm{A}, \mathrm{R}_{\mathrm{N}} / 2=$ $0.78 \Omega$, and $\Delta V=1.8 \mu V$ and $15 \mu V$ for type $A$ and type $B$ SQUIDs, respectively. The standard deviation in $2 \mathrm{I}_{c}$ corresponds to about $\pm 30 \%$ of the average value and in $R_{N} / 2$ to about $\pm 20 \%$ for both type $A$ and type $B$ devices. For the type A devices, the standard deviation in $\Delta \mathrm{V}$ corresponds to about $\pm 20 \%$. We note, however, that the standard deviation in

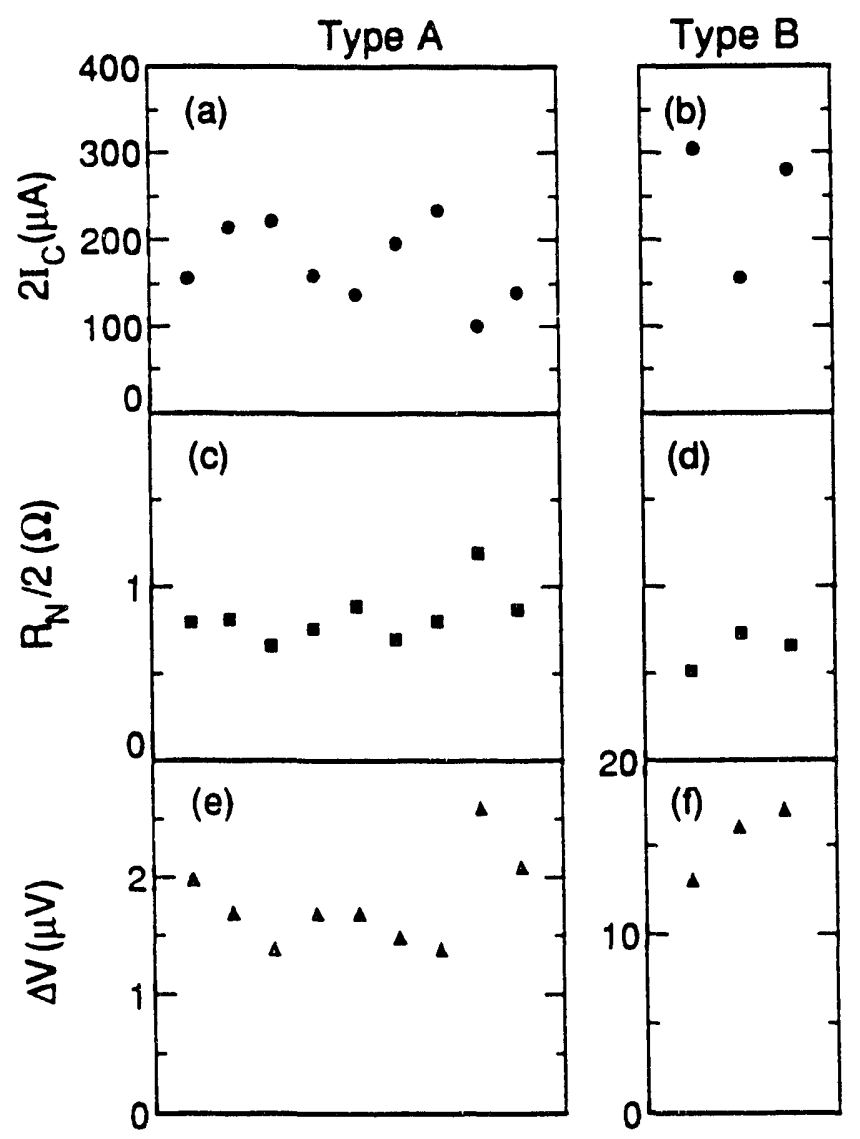

Fig. 2 Critical current (a) and (b), asymptotic resistance (c) and (d), and peak-to-peak voltage modulation, $\Delta \mathrm{V}$, (e) and (f) at $77 \mathrm{~K}$ for 12 SQUIDs on a single bicrystal. Abscissa enumerates the SQUIDs.
$I_{C} R_{N}$ corresponds to about $\pm 18 \%$, suggesting that some of the scatter in $I_{0}$ and $R_{N}$ may well be due to small variations in the widths of the junctions. An additional variation in $\mathrm{I}_{\mathbf{c}}$ may arise from the flux trapped in the junctions. The scatter in $\Delta V / R_{N}$ is only $\pm 11 \%$ in type $A$ and $\pm 9 \%$ in type $B$, implying that the modulation depth in critical current is constant to this level.

All SQUIDs of the same type have remarkably similar effective magnetic flux capture areas. For the type A SQUIDs this was $\Phi_{0} / 0.14 \mu \mathrm{T}=1.5 \times 10^{-8} \mathrm{~m}^{2}$; neglecting the slit, we expect an effective area [11] $A_{s}$ of $25 \mu \mathrm{m} \times 250 \mu \mathrm{m}=$ $6.3 \times 10^{-9} \mathrm{~m}^{2}$. Thus the slit increases the effective area by a factor of about 2.5. For the type B SQUIDs, the measured effective area for the $250 \mu \mathrm{m}$ washer was $\Phi_{0} 10.48 \mu \mathrm{T}=4.3 \mathrm{x}$ $10^{-9} \mathrm{~m}^{2}$ compared with the predicted value, neglecting the slits, of $6.3 \times 10^{-9} \mathrm{~m}^{2}$, while for the $500 \mu \mathrm{m}$ washer the measured value was $\Phi_{0} / 0.32 \mu \mathrm{T}=6.5 \times 10^{-9} \mathrm{~m}^{2}$ compared with the predicted value of $1.25 \times 10^{-8} \mathrm{~m}^{2}$. Thus, for the type B SQUIDs the presence of the slits reduces the effective area by $32 \%$ and $48 \%$ for the $250 \mu \mathrm{m}$ and $500 \mu \mathrm{m}$ washer, respectively.

For one type B SQUID that we studied in detail, the measured flux-to-voltage transfer coefficient was $\mathrm{dV} / \mathrm{d} \Phi=(65$ $\pm 10) \mu V / \Phi_{0}$. From the measured dimensions of the inner hole, $d^{2}=26 \mu \mathrm{m} \times 26 \mu \mathrm{m}$, we estimate an inductance [12] $\mathrm{L}=$ $1.25 \mu_{0} d=41 \mathrm{pH}$. Using the measured asymptotic resistance $R_{N}=1.28 \Omega$, we expect [13] dV/d $\Phi-65 \mu \mathrm{V} / \Phi_{0}$, an agreement that is excellent but undoubtedly fortwitous, given the uncertainties in the parameters. In the case of the type A SQUIDs, however, the transfer coefficients are much smaller, ranging up to $6 \mu \mathrm{V} / \Phi_{0}$ in a device with $R_{N}=2.4 \Omega$. Estimating the inductance of the slit as $L_{3}=\mu_{0} l / 2$ for a length $\ell=152 \mu \mathrm{m}$ we find $L_{s}=95 \mathrm{pH}$ for a total SQUID inductance of about $135 \mathrm{pH}$. Thus, we would expect $\mathrm{dV} / \mathrm{d} \Phi \approx$ $37 \mu \mathrm{V} / \Phi_{0}$, considerably larger than the observed transfer coefficient. The reason for the reduced response of the type A SQUIDs is not clear, one explanation is that the inductance associated with the slit is higher than anticipated.

Having determined the parameters of our SQUIDs, we measured the flux noise in one of them at $77 \mathrm{~K}$, first using a static current bias and a flux modulation frequency of $100 \mathrm{kHz}$. The flux noise power spectrum, $S_{\Phi}(f)$, and the noise energy, $\varepsilon(f) \equiv S_{\Phi}(f) / 2 L$, are shown as the upper trace of Fig. 3 for the type B SQUID discussed in the paragraph above with an outer dimension of $500 \mu \mathrm{m}$. At frequencies above $5 \mathrm{kHz}$ the spectral density becomes white with a value of $S_{\Phi}(10 \mathrm{kHz})=3.5 \mathrm{x}$ $10^{-11} \Phi_{0}^{2} \mathrm{~Hz}^{-1}$, corresponding to $\varepsilon(10 \mathrm{kHz})=1.8 \times 10^{-30}$ $\mathrm{JHz}^{-1}$. By comparison, using $\mathrm{R}_{\mathrm{N}}=1.3 \Omega$ and $\mathrm{L}=41 \mathrm{pH}$, we predict [13] $\varepsilon \approx 9 \mathrm{kB}_{\mathrm{B}} \mathrm{TL} / \mathrm{R} \approx 3 \times 10^{-31} \mathrm{JHz}^{-1}$, a factor of 6 smaller. Part, although not all, of this discrepancy, may be explained by noise generated in the contact resistance to the SQUID.

Between $0.1 \mathrm{~Hz}$ and $1 \mathrm{kHz}$, the power spectrum scales closely as $\mathrm{V} / \mathrm{f}$. In an attempt to reduce this noise, we used a bias current reversal scheme. [14] We divide the $100 \mathrm{kHz}$ square wave modulation signal from the flux-locked loop using a digital counting circuit to produce a synchronous 


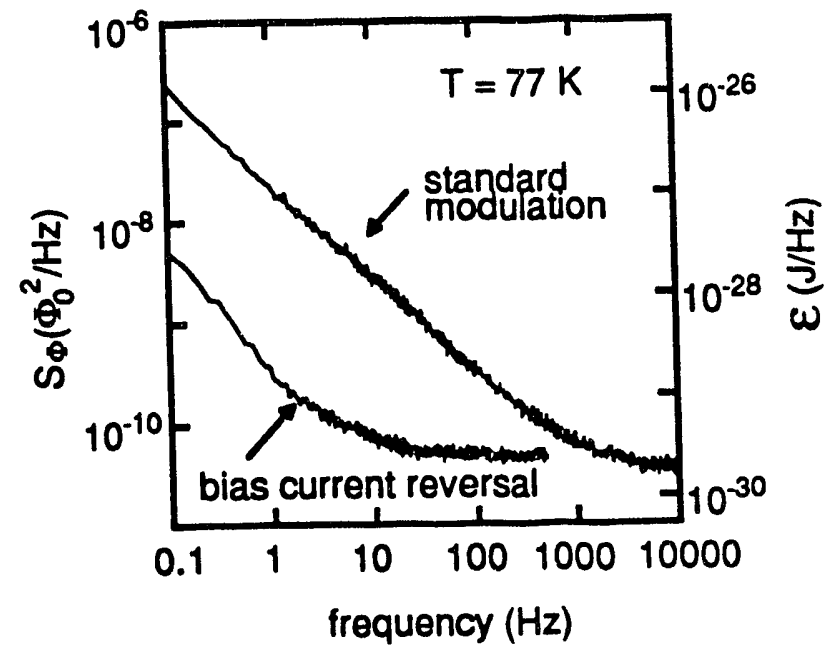

Fig. 3 Flux noise spectral density, $S_{\Phi}(f)$, and noise energy, $\varepsilon(f)$, for type B SQUID (500um outer dimension) at 77K. Upper trace is for conventional flux-locked loop with static current bias, lower trace is for bias reversal scheme.

$2 \mathrm{kHz}$ square wave that reverses the bias current and simultaneously shifts the flux in the SQUID by $\Phi_{0} / 2$. The resultant noise spectral density (lower trace in Fig. 3) reduced by two orders of magnitude at $1 \mathrm{~Hz}$. This lar'se reduction demonstrates that the $1 / f$ noise in the upper trace of Fig. 3 arises from critical current fluctuations rather than from flux noise. The bias reversal scheme has caused the white noise to increase slightly, to $2.5 \times 10^{-30} \mathrm{JHz}^{-1}$. The residual noise, $S_{\Phi}(1 \mathrm{~Hz})=2.9 \times 10^{-10} \Phi_{0}^{2} \mathrm{~Hz}^{-1}$, may be due to flux noise within the body of the SQUID, or may represent the limit to which we have thus far been able to suppress the critical current fluctuations. We note that Koch et al. [4] have previously reported a similar degree of noise reduction in a bicrystal SQUID; however, the residual noise at $1 \mathrm{~Hz}$ in their case was considerably higher than in our devices. At the time of writing, our measured noise energy at $1 \mathrm{~Hz}, 1.5 \times 10^{-29}$ $\mathrm{JHz}^{-1}$, is the lowest value we are aware of in a ds SQUID operating at $77 \mathrm{~K}$.

\section{MAGNETOMETERS}

To make a sensitive magnetometer from our SQUIDs, it is essential to increase the flux capture area with a suitable flux transformer. We have coupled the low noise type B SQUID discussed above ( $500 \mu \mathrm{m}$ washer) in a flip-chip arrangement to a thin-film flux transformer [6] with a 5-turn input coil and a $10 \mathrm{~mm}$ pick-up loop with an approximate area of $81 \mathrm{~mm}^{2}$. The estimated inductance of the pick-up loop, $L_{p}=20 \mathrm{nH}$, greatly exceeds the inductance of the input coil $\mathrm{L}_{\mathrm{i}}=\operatorname{lnH}$. In this limit, the magnetic field gain of the transformer is approximately $\left(A_{p} / A_{s}\right) \alpha\left(L L_{i}\right) / / 2 / L_{p}$. The measured gain was about 60 , indicating a magnetic coupling coefficient $\alpha \approx$ 0.5. The spectral density of the noise is plotted in Fig. 4. At $10 \mathrm{kHz}$ the noise of the magnetometer corresponded to approximately $36 \mathrm{fT} \mathrm{Hz}^{-1 / 2}$. However, at frequencies below a few kilohertz the noise power scaled as $\mathrm{V} / \mathrm{f}$. The magnitude of this noise was not measurably reduced by our bias reversal

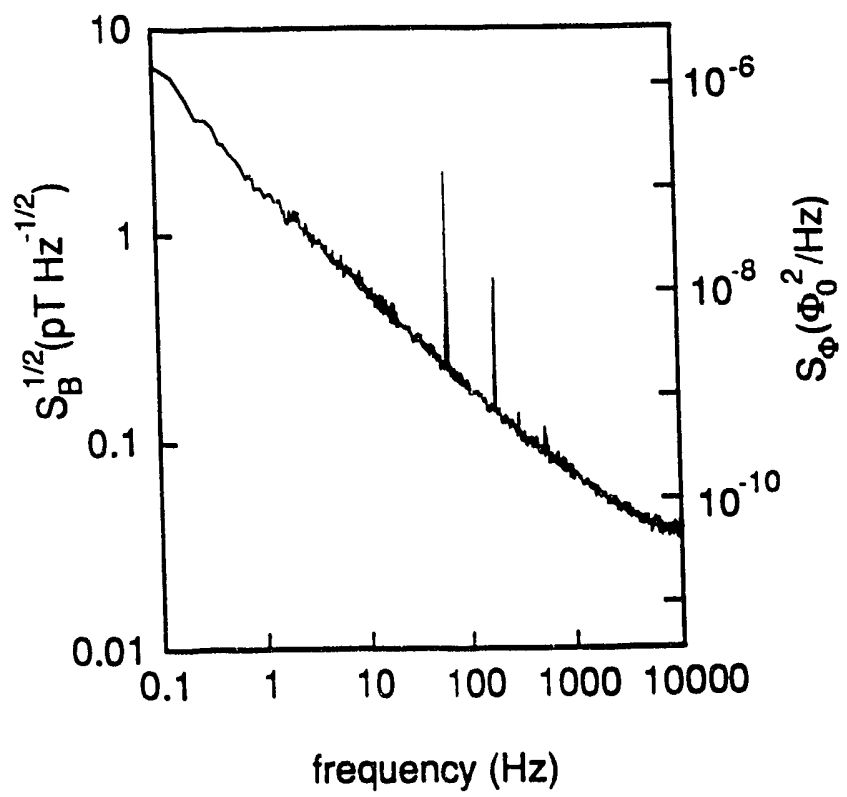

Fig. 4 Rms magnetic field resolution, $S_{B}^{1 / 2}(f)$, and flux noise spectral density, $S_{\Phi}(f)$, vs. frequency for 5-turn flux transformer coupled to the type B SQUID of Fig. 3. Low frequency noise was not reduced by bias reversal scheme.

scheme, indicating that it arose from flux noise in the films of the transformer. The magnetic field noise at $1 \mathrm{~Hz}$ was $1.7 \mathrm{pT} \mathrm{Hz}-1 / 2$. This high noise level stresses the need to be able to deposit and pattem multilayer films while maintaining very high film quality and concomitantly very low levels of flux noise. We note that the number of turns on the input coil of the flux transformer was below optimum for the size of the pick-up loop. A properly optimized transformer with the same pick-up loop would have had a white noise of approximately $10 \mathrm{fT} \mathrm{Hz}^{-1 / 2}$.

Finally, we have made a simple magnetometer in which a loop of YBCO, deposited and patterned along with the SQUID, is coupled directly to the body of a type A SQUID (Fig. 5). The magnetic field gain of such a device is approximately $\left(L / L_{p}\right)\left(A_{p} / A_{s}\right)$. Estimating $L_{p}=15 n H, A_{p}=$ $56 \mathrm{~mm}^{2}, \mathrm{~L}=135 \mathrm{pH}$ and using the measured value $A_{s}=1.5$ $x 10^{-2} \mathrm{~mm}^{2}$, we expect a gain of about 34 . The measured gain at $77 \mathrm{~K}$ was only 22 , approximately two-thirds of the predicted value. We measured the noise of one device at $77 \mathrm{~K}$ and found a white noise of $245 \mathrm{fT} \mathrm{Hz}^{-1 / 2}$, corresponding to a flux noise of $38 \mu \Phi_{0} \mathrm{~Hz}^{-1 / 2}$ which is expected for a type $A$ SQUID. The $1 / f$ noise was unfortunately very high, increasing the magnetic field noise at $1 \mathrm{~Hz}$ to $9.5 \mathrm{pT} \mathrm{Hz}^{-1 / 2}$, a value which was not reduced by bias current reversal. This level of flux noise in our films is unusually high, and we expect future devices to have much lower noise. Although such a device cannot compete in sensitivity with an optimized multitum flux transformer, its simplicity is very appealing. The design could be readily adapted to a planar gradiometer that might well have adequate sensitivity for certain applications, for example, non-destructive evaluation. 


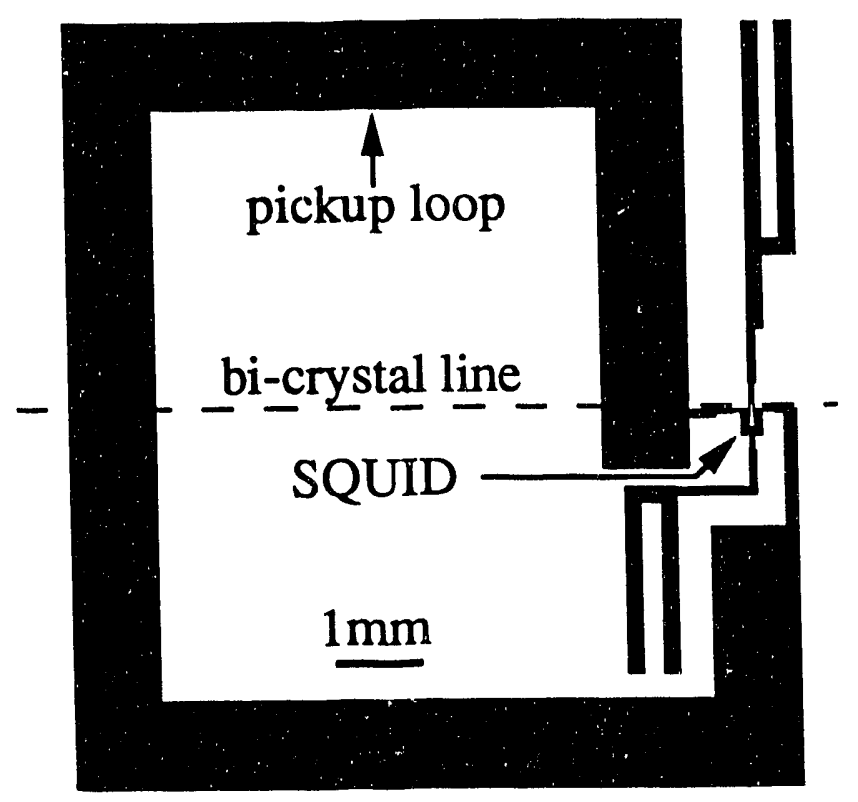

Fig. 5 Configuration of single-turn magnetometer. The pick-up loop connects to the SQUID body on either side of the slit, just below the two junctions. The four vertical lines extending from the SQUID are contact pads.

\section{REFERENCES}

[1] G. Friedl, M. Vildic, B. Roas, D. Uhl, F. Bommel, M. Romheld, B. Hillenbrand, B. Stritzker and G. Daalmans, "Low 1/f noise single-layer $\mathrm{YBa}_{2} \mathrm{Cu}_{3} \mathrm{O}_{\mathrm{X}}$ dc SQUID at 77 K," Appl. Phys. Lett., vol. 60, pp. 3048-3050, June 1992.

[2] Y. Zhang, H.-M. Mück, K. Herrmann, J. Schubert, W. Zander, A. I. Braginski and C. Heiden, "Low noise $\mathrm{YBa}_{2} \mathrm{Cu}_{3} \mathrm{O}_{\mathrm{x}}$ if SQUID magnetometer," Appl. Phys. Lett., vol. 60, pp. 645-647, February 1992.

[3] R. Gross, P. Chaudhari, M. Kawasaki, M. B. Ketchen and $A$. Gupta, "Low noise $\mathrm{YBa}_{2} \mathrm{Cu}_{3} \mathrm{O}_{7}-\delta$ grain boundary junction dc SQUIDs," Appl. Phys. Lett., vol. 57, pp. 727-729, August 1990; M. Kawasaki, P. Chaudhari, T. H. Newman and A. Gupta, "Submicron $\mathrm{YBa}_{2} \mathrm{Cu}_{3} \mathrm{O}_{7}-\delta$, grain boundary junction de SQUIDs," Appl. Phys. Lett., vol. 58, pp. 2555-2557, June 1991.

[4] R. H. Koch, W. Eidelloth, B. Oh, R. P. Robertazzi, S. A. Andrek and W. J. Gallagher, "Identifying the source of $1 / f$ noise in SQUIDs made from high-temperature superconductors," Appl. Phys. Lett., vol. 60, pp. 507509, January 1992.

[5] M. S. Dilorio, S. Yoshizumi, M. Maung, K. Y. Yang, J. Zhang and N. Q. Fan, "Manufacturable low-noise SQUIDs operating in liquid nitrogen," Nature, vol. 354, pp. 513-515, December 1991.

[6] A. H. Miklich, J. J. Kingston, F. C. Wellstood, John Clarke, M. S. Colclough, K. Char and G. Zaharchuk, "Sensitive $\mathrm{YBa}_{2} \mathrm{Cu}_{3} \mathrm{O}_{7-x}$ thin-film magnetometer," Appl. Phys. Lett., vol. 59, pp. 988-990, August 1991.

[7] M. Kawasaki, P. Chaudhari and A. Gupta, "1/f Noise in $\mathrm{YBa}_{2} \mathrm{Cu}_{3} \mathrm{O}_{7-\delta}$ superconducting bicrystal grain-boundary junctions," Phys. Rev. Lett., vol. 68, pp. 1065-1067, February 1992.

[8] A. H. Miklich, John Clarke, M. S. Colclough and K. Char, "Flicker (V/) noise in biepitaxial grain boundary junctions of $\mathrm{YBa}_{2} \mathrm{Cu}_{3} \mathrm{O}_{7-x}$, , Appl. Phys. Lett, vol. 60, pp. 1899-1901, April 1992.

[9] M. J. Ferrari, Mark Johnson, Frederick C. Wellstood, John Clarke, P. A. Rosenthal, R. H. Hammond and M. $R$. Beasley, "Magnetic flux noise in thin-film rings of $\mathrm{YBa}_{2} \mathrm{Cu}_{3} \mathrm{O}_{7}-\delta$," Appl. Phys. Lett., vol. 53, pp. 695697, August 1988.

[10] Wako Bussan Co., Ltd, Tokyo.

[11] M. B. Ketchen, W. J. Gallagher, A. W. Kleinsasser, S. Murphy and J. R. Clem, "DC SQUID flux focuser," in Proceedings of SQUID '85, Berlin, Germany, June 1985, pp. 865-871.

[12] J. M. Jaycox and M. B. Ketchen, "Planar coupling scheme for ultra low noise SQUIDs," IEEE Trans. Magn., MAG-17, pp. 400-403 January 1981.

[13] C. D. Tesche and J. Clarke, "DC SQUID: Noise and optimization," J. Low Temp. Phys., vol. 29, pp. 301-331 November 1977.

[14] R. H. Koch, J. Clarke, W. M. Goubau, J. M. Martinis, C. M. Pegrun and D. J. Van Harlingen, "Flicker (1/f) noise in tunnel junction de SQUIDs," J. Low Temp. Phys., vol. 51, pp. 207-224, April 1981. 

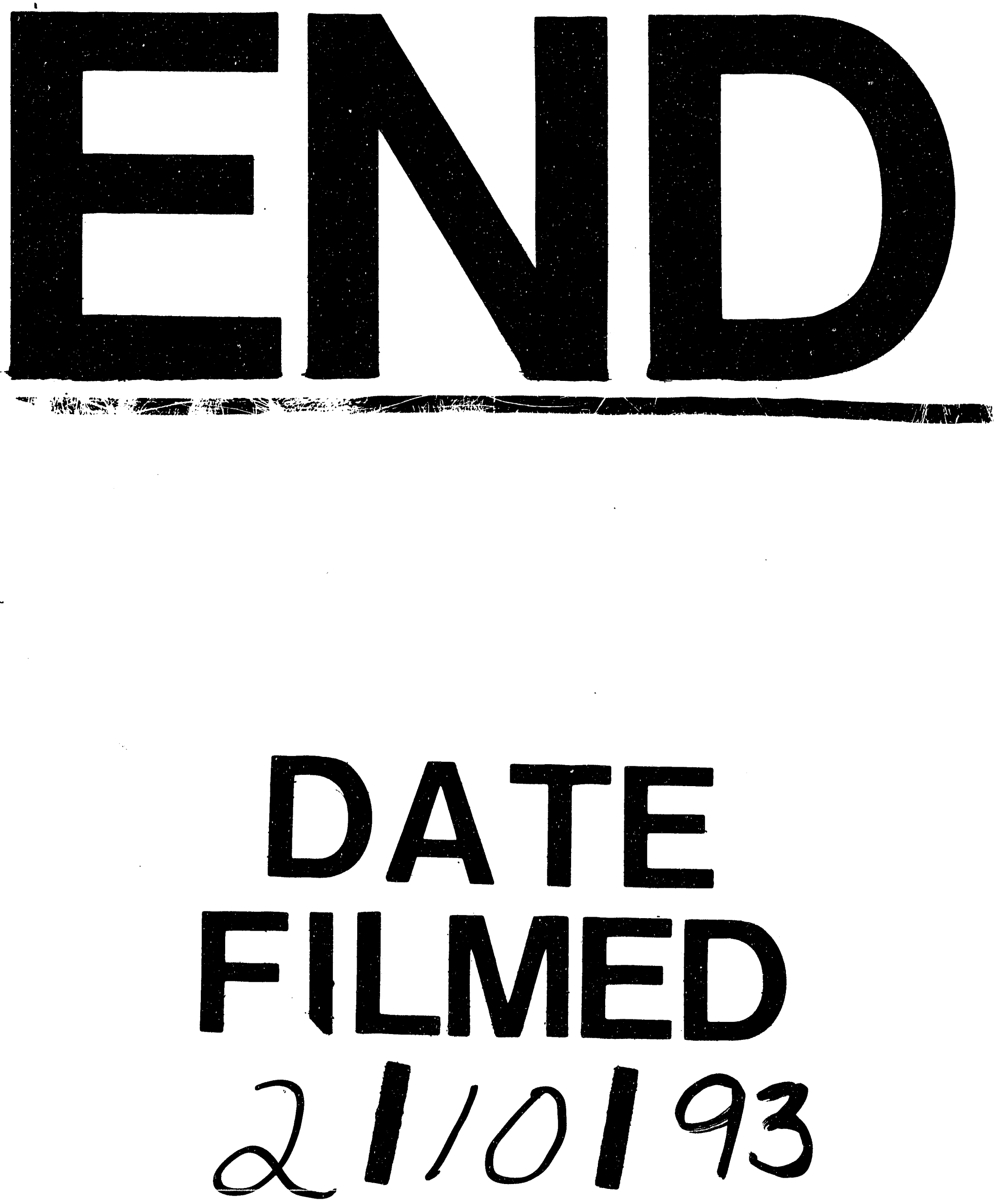


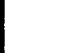

.
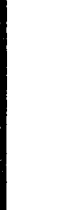